\title{
Preparation of gallium sulfide nanosheets by liquid exfoliation and their application as hydrogen evolution catalysts
}

\author{
Andrew Harvey, ${ }^{1,2}$ Claudia Backes, ${ }^{1,2}$ Zahra Gholamvand, ${ }^{1,2}$ Damien Hanlon, ${ }^{1,2}$ David \\ McAteer, ${ }^{1,2}$ Hannah C. Nerl, ${ }^{1,2,3}$ Eva McGuire, ${ }^{1,2,3}$ Andrés Seral-Ascaso, ${ }^{1,2,3}$ Quentin M. \\ Ramasse, ${ }^{4}$ Niall McEvoy, ${ }^{1,3}$ Sinéad Winters, ${ }^{1,3}$ Nina C. Berner, ${ }^{1,3}$ David McCloskey, ${ }^{1,2}$ John \\ F. Donegan, ${ }^{1,2}$ Georg S. Duesberg, ${ }^{1,3}$ Valeria Nicolosi ${ }^{1,2,3}$ and Jonathan N. Coleman ${ }^{1,2 *}$ \\ ${ }^{1}$ CRANN \& AMBER, Trinity College Dublin, Dublin 2, Ireland \\ ${ }^{2}$ School of Physics, Trinity College Dublin, Dublin 2, Ireland \\ ${ }^{3}$ School of Chemistry, Trinity College Dublin, Dublin 2, Ireland \\ ${ }^{4}$ SuperSTEM Laboratory, STFC Daresbury Campus, Daresbury, WA4 4AD, United Kingdom \\ *colemaj@tcd.ie
}

\begin{abstract}
Here we demonstrate the production of large quantities of gallium sulfide ( $\mathrm{GaS}$ ) nanosheets by liquid exfoliation of layered GaS powder. The exfoliation was achieved by sonication of the powder in suitable solvents. The variation of dispersed concentration with solvent was consistent with classical solution thermodynamics and showed successful solvents to be those with Hildebrand solubility parameters close to $21.5 \mathrm{MPa}^{1 / 2}$. In this way, nanosheets could be produced at concentrations of up to $\sim 0.2 \mathrm{mg} / \mathrm{ml}$ with lateral sizes and thicknesses of $50-1000$ $\mathrm{nm}$ and 3-80 layers, respectively. The nanosheets appeared to be relatively defect free although oxygen was observed in the vicinity of the edges. Using controlled centrifugation techniques, it was possible to prepare dispersions containing size-selected nanosheets. Spectroscopic measurements showed the optical properties of the dispersions to vary strongly with nanosheet size, allowing the elucidation of spectroscopic metrics for in-situ estimation of nanosheet size and thickness. These techniques allow the production of nanosheets with controlled sizes which will be important for certain applications. To demonstrate this, we prepared films of GaS nanosheets of three different sizes for use as hydrogen evolution electrocatalysts. We found a clear correlation between performance and size showing small nanosheets to be more effective. This is consistent with the catalytically active sites residing on the nanosheet edges.
\end{abstract}




\section{Introduction}

Over the last few years, liquid phase exfoliation (LPE) has become an increasingly important technique for the production of two-dimensional nanomaterials. ${ }^{1-3}$ This method involves the delamination of layered crystals, usually by exposure to ultra sonication ${ }^{4,5}$ or high shear rates, ${ }^{6-9}$ to form large quantities of two-dimensional nanosheets. These are then stabilized against aggregation through interaction with appropriate solvents, ${ }^{1,10-20}$ or ionic liquids ${ }^{21}$ or by coating with surfactants ${ }^{4,22-32}$ or adsorbed polymer chains. ${ }^{33-36}$ The resultant nanosheets range in lateral size from $<100 \mathrm{~nm}$ to $>2000 \mathrm{~nm}$, depending on the material in question. ${ }^{37,}{ }^{38}$ The nanosheet thickness tends to be broadly distributed between approximately one and 10 layers with monolayer contents of tens of percent achievable. ${ }^{6,37}$ That nanosheets can be stably dispersed in liquids greatly facilitates further processing. For example nanosheets can be sizeselected by controlled centrifugation ${ }^{23,37-41}$ or chemically modified via functionalization protocols. ${ }^{42-44}$ In addition, the dispersions can easily be formed into thin films or mixed with other materials to form composites. ${ }^{4} 6$ This has enabled the use of liquid exfoliated nanosheets in a range of applications from electrocatalysis, ${ }^{37,45,46}$ to composite reinforcement ${ }^{47-51}$ to inkjetprinted devices. ${ }^{52,53}$ Additional advantages of this technique are that it is low-cost, relatively straightforward to implement and can easily be scaled up to produce very large quantities of nanosheets.

Liquid phase exfoliation was first used to produce graphene from graphite in 2008., 54 Subsequent work has seen the scale up and commercialization of this method as a graphene production technique. ${ }^{6}$ Possibly the greatest strength of this technique is its versatility. Liquid phase exfoliation has been used to produce nanosheets from a wide range of layered crystals beyond graphite including; boron nitride; transition metal dichalcogenides, from $\mathrm{MoS}_{2}$ to $\mathrm{WTe}_{2} ; \mathrm{MoO}_{3}$ and black phosphorus. ${ }^{2,4,5,11,23,39,40,50,55-58}$ However, because there are hundreds of different types of layered crystals, ${ }^{2}$ this material set represents only the tip of the iceberg of materials that could be exfoliated by LPE. We believe it is critically important to use LPE to exfoliate yet untested layered crystals and to produce new types of nanosheets. These new materials will be of interest both for basic studies and because of their potential for use in a range of new applications.

The family of III-VI layered semiconductors is a good example of a largely untapped source of two-dimensional materials. These materials generally exist in the form $\mathrm{MX}$ where $\mathrm{M}=\mathrm{Ga}$, In and $\mathrm{X}=\mathrm{S}, \mathrm{Se}, \mathrm{Te}$ (see figure $1 \mathrm{~A}$ ) although other stoichiometries also exist. ${ }^{59}$ These materials 
are wide bandgap semiconductors and are of interest for a range of applications from electrochemistry ${ }^{60}$ to optoelectronics, ${ }^{61-63}$ gas sensing ${ }^{64}$ and nonlinear optics. ${ }^{65}$ Over the last thirty years a number of papers have studied the properties of such layered crystals with common examples being GaSe, InTe and the related compound $\mathrm{In}_{2} \mathrm{Se}_{3} .{ }^{65-67}$ However, work on exfoliation has only started recently with a number of papers reporting production of few-layer InSe and GaSe by mechanical cleavage or chemical exfoliation as well as vapor phase growth of thin layers. ${ }^{59,68-71}$ Such studies have found these exfoliated materials to be of interest for applications such as photodetectors and in non-linear optics. ${ }^{59,68-72}$

A typical representative example of this family is gallium sulfide, GaS. Although found in a variety of structures such as nanobelts and tubes, ${ }^{73}$ this material is most commonly encountered as a layered crystal and is particularly attractive due to its relatively low cost. Although a number of papers have studied the layered form of $\mathrm{GaS},{ }^{62,63}, 74$ work on its exfoliation to give nanosheets is in the very early stages. Exfoliation has up to now only been achieved by micromechanical cleavage ${ }^{61,64,75}$ which suffers from low throughput and can only produce material quantities suitable for fundamental studies. However, it is clear that the properties of exfoliated gallium sulfide are interesting and differ from the bulk form. ${ }^{60,61,64,75}$ For example, exfoliated nanosheets of GaS have been used to fabricate sensitive photodetectors. ${ }^{61,64}$ In addition, it is likely that $\mathrm{GaS}$ is useful in applications also beyond (opto)electronics. Gallium sulfide produced by atomic layer deposition has been combined with carbon nanotubes to produce high performance anodes in Li ion batteries. ${ }^{60}$ However, for such materials to be competitive in applications such as battery electrodes, large quantities would be needed. Applications such as this highlight the need to develop a scalable method to produce nanosheets of gallium sulfide and related materials.

Herein we report that layered III-VI semiconductors can be exfoliated in solvents by bath sonication. The resultant dispersions contain nanosheets which are of high quality and appear to be defect-free except for an increased oxygen content at the vicinity of edges. In addition, spectroscopic properties strongly vary as a function of nanosheet size allowing us to establish quantitative metrics to determine mean length and thickness spectroscopically. This facilitates the production of dispersions with well-defined nanosheet sizes and so specific properties. These were subsequently used to fabricate GaS electrodes for electrocatalysis of hydrogen production. Strong size effects are found with smaller nanosheets performing better. 


\section{Results and Discussion}

\section{Evidence of exfoliation and basic characterization}

In this work, we study the exfoliation of layered $\mathrm{GaS}$ in a number of solvents. We chose $\mathrm{GaS}$ as a representative member of the family of layered III-VI semiconductors (structure see Fig. 1A) in part due to its commercial availability. To perform the exfoliation, we added GaS powder to a variety of solvents and agitated using an ultrasonic bath for a fixed period. The dispersions were then centrifuged to remove unexfoliated material. In most cases, we obtained pale yellow colored liquids such as the one shown in figure $1 \mathrm{~B}$.

In order to confirm the exfoliation of the layered powder to $2 \mathrm{D}$ nanosheets, we performed low-resolution transmission electron microscopy (TEM) imaging (Fig. 1C). These measurements showed all dispersions tested to contain large quantities of electron-transparent 2D nanosheets. In addition, bright field (Fig. 1D) and high angle annular dark field dark (HAADF) (Fig. 1E) scanning transmission electron microscopy (STEM) imaging confirmed the $2 \mathrm{D}$ crystal lattice to be intact.

Typically, the dispersed concentration of stable, exfoliated nanomaterials has been estimated from measurements of the optical extinction at a given wavelength ${ }^{4-6,39}$ (the extinction, Ext, is defined via the optical transmittance: $T=10^{-E x t}$ ). The measured extinction spectrum of a typical GaS dispersion is plotted as the black curve in figure 1F (solvent isopropanol, initial $\mathrm{GaS}$ concentration $\mathrm{c}_{\mathrm{i}}=45 \mathrm{~g} / \mathrm{L}$, sonication time $\mathrm{t}_{\mathrm{s}}=6 \mathrm{~h}$, centrifugation time $\mathrm{t}_{\mathrm{cf}}=180 \mathrm{~min}$ at $2.5 \mathrm{krpm}$ equivalent to $665 \mathrm{~g}$ ). This plot shows a near monotonic increase with decreasing wavelength with no features of note, bar a small peak at $315 \mathrm{~nm}$. As with dispersions of other 2D materials, such curves are relatively featureless because extinction spectra of dispersed nano-objects contain a significant contribution from scattering ( $\mathrm{Sca}$ ) in addition to the actual absorbance (Abs) of the material (NB: extinction, absorbance and scattering are related by $\operatorname{Ext}(\lambda)=\operatorname{Abs}(\lambda)+\operatorname{Sca}(\lambda))$.

The absorbance and scattering components can be differentiated from the overall extinction spectra using an integrating sphere ${ }^{37}$ as shown in figure $1 \mathrm{~F}$. The absorbance is very low in the high wavelength regime, becoming appreciable only for $\lambda<400 \mathrm{~nm}$ consistent with the semiconducting nature of GaS. This data does indeed show a significant scattering component, particularly in the non-resonant regime $(\lambda>400 \mathrm{~nm})$. The shape of the spectra will be discussed in more detail below. Using extinction spectra to estimate dispersed concentration has been 
complicated by the recent realization that both absorbance and scattering coefficients are generally dependent on nanosheet size. ${ }^{37,76,77}$ The wavelength used has to be chosen with care and requires an understanding of the size-dependent extinction coefficients as discussed below. In the case of $\mathrm{GaS}$, we found the extinction coefficient at $365 \mathrm{~nm}$ to be relatively nanosheet size independent with a value of $\varepsilon_{365 n m} \approx 3654 \mathrm{~L} \mathrm{~g}^{-1} \mathrm{~m}^{-1}$. This allows the estimation of the dispersed nanosheet concentration, $\mathrm{C}$, using $\operatorname{Ext}_{365 \mathrm{~nm}}=\varepsilon_{365 \mathrm{~nm}} \mathrm{Cl}$ (1 is the cell length).

To identify the most appropriate solvents for the liquid exfoliation of $\mathrm{GaS}$ and to investigate the exfoliation/stabilization mechanism, we have sonicated and centrifuged the powder in 15 solvents to produce nanosheets under identical processing conditions (see methods). We measured optical extinction spectra for each dispersion, using Ext365nm as a measure of the dispersed concentration. In this way we found large variations in dispersed concentration among the solvents studied, with the highest nanosheet contents found for amide solvents such as N-methyl-2-pyrrolidone (NMP) and N-cyclohexyl-2-pyrrolidone (CHP). To understand the exfoliation/stabilization mechanism, we plot Ext $365 \mathrm{~nm} / 1$ versus the solvent Hildebrand solubility parameter $\delta$ s in figure $1 \mathrm{G}$. The concentrations of exfoliated GaS, estimated using $\varepsilon_{365 n m} \approx 3654$ $\mathrm{L} \mathrm{g}^{-1} \mathrm{~m}^{-1}$, are shown on the right axis. We find a clear and well defined peak centered at around 21.5 $\mathrm{MPa}^{1 / 2}$ very similar to studies on other $2 \mathrm{D}$ nanomaterials. ${ }^{4,5,10,39}$

According to classical solution thermodynamics, in the simplest case, ${ }^{55,78}$ the saturated concentration, $\mathrm{C}$, of 2D solutes, such as the GaS nanosheets studied here is given by

$$
C \propto \exp \left[-\frac{v_{N S}}{3 k T}\left(\delta_{S}-\delta_{N S}\right)^{2}\right]
$$

where $\delta_{N S}$ represents the Hildebrand parameter of the 2-dimensional solute, $v_{N S}$ represents the unit volume of the solute while the factor of three stems from the solute dimensionality. ${ }^{78}$ The dashed line in figure $1 \mathrm{G}$ (inset) is a fit to equation (1) and shows very good agreement with the experimental data, further confirming the validity of solution thermodynamics in the case of nanomaterial dispersions. The data implies the Hildebrand solubility parameter of GaS nanosheets to be $\sim 21.5 \mathrm{MPa}^{1 / 2}$, close to values of $23, \sim 22.5,22,21$ and reported recently for Graphene, ${ }^{10} \mathrm{BN},{ }^{4} \mathrm{MoS}_{2}\left(\right.$ ref $\left.^{55}\right)$ and $\mathrm{MoO}_{3}\left(\right.$ ref $\left.^{39}\right)$, respectively. We note that analysis in terms of Hildebrand parameters has been shown to be equivalent to analysis in terms of surface energies. ${ }^{78}$ Essentially, this means successful solvents are those with surface tensions close to $40 \mathrm{~mJ} / \mathrm{m}^{2}$. Taken together, this means that, as has been observed previously for the materials 
mentioned above, liquid phase exfoliation of $\mathrm{GaS}$ is most favorable in solvents whose interaction with the nanosheets is such that the energy of exfoliation is minimized. ${ }^{5}$

Even though we found empirically that NMP and CHP are the most suitable solvents in terms of dispersed concentration, we have used 2-propanol (IPA) for the remainder of the study (these solvents are marked in red in figure $1 \mathrm{G}$ ). Although the concentration of GaS which can be exfoliated in IPA is only a quarter that achievable in NMP, use of IPA brings significant advantages in processing and analysis due to its low toxicity and low boiling point. We fully optimized the exfoliation conditions in terms of centrifugation rate and time, sonication time and initial GaS concentration as presented in the SI figure S1. We found the following parameters to be optimized for the production of standard dispersions of exfoliated GaS in IPA: initial GaS concentration $\mathrm{C}_{\mathrm{i}}=45 \mathrm{~g} / \mathrm{L}$, sonication time $\mathrm{t}_{\mathrm{s}}=6 \mathrm{~h}$, centrifugation rate $f=2.5 \mathrm{krpm}$ (equivalent to $665 \mathrm{~g}$ ), centrifugation time $\mathrm{t}_{\mathrm{CF}}=180 \mathrm{~min}$. Under these circumstances, we could produce dispersions with concentrations of roughly $\mathrm{C}=0.22 \mathrm{~g} / \mathrm{L}$ of dispersed nanosheets.

It is important to confirm that the exfoliated $\mathrm{GaS}$ is pristine and defect free. For this purpose, samples were subjected to Raman and X-ray photoelectron spectroscopies (XPS). Figure $1 \mathrm{H}$ shows the Raman spectrum (excitation wavelength $532 \mathrm{~nm}$, mean of 20 spectra) of a filtered film of the standard dispersion. The films are homogenous in appearance as evidenced by scanning electron microscopy (SEM) (inset in figure $1 \mathrm{H}$ ). The characteristic lattice vibrations of $\mathrm{GaS}$ are detected at 195,300 and $370 \mathrm{~cm}^{-1}$ as assigned in the figure. ${ }^{61,74,75}$ Only minor contributions from other materials are observed such as the $v_{1}\left(\mathrm{~A}_{1}\right)$ mode of the $\mathrm{GaS}_{4}$ molecular unit as typically found in $\mathrm{Ga}_{2} \mathrm{~S}_{3}$ which we see at $\sim 240 \mathrm{~cm}^{-1} .{ }^{79,80}$ The fitted XPS core level spectra (Ga 3d in Fig 1I and S 2p in Fig. 1J) further confirm that the exfoliated material is $\mathrm{GaS}$ with minor contributions from $\mathrm{Ga}_{\mathrm{x}} \mathrm{O}_{\mathrm{y}}$. We note that such oxide species were also found in the starting powder (Fig. S2). In addition, all XPS peaks measured for the starting powder are significantly broadened in terms of full width at half maximum of the fit components, suggesting a lower degree of order and/or purity in the starting powder compared to the exfoliated material. This shows that the sonication and centrifugation partially purifies the starting material by removal of unwanted, components such as non-layered impurities. This can be illustrated by the presence of significant amounts of $\mathrm{Ga}_{2} \mathrm{~S}_{3}$ in the starting powder as shown by X-ray diffraction (XRD) (Fig. S3) and Raman spectroscopy (Fig. S4). Since $\mathrm{Ga}_{2} \mathrm{~S}_{3}$ is not layered, it is not exfoliated and therefore largely removed during centrifugation. 


\section{Electron energy loss spectroscopy}

Since XPS shows that oxides are present in both GaS powder and exfoliated nanosheets (Fig. 1I and S2) and virtually nothing is known about the long term stability of GaS in the exfoliated state, it is important to track where oxides species reside. This is particularly crucial in light of recent investigations on exfoliated black phosphorus nanosheets which have shown significant degradation under exposure to ambient conditions. ${ }^{81-83}$ To gain insights into potential oxidation of GaS, we have analyzed liquid exfoliated nanosheets by STEM imaging and electron energy loss spectroscopy (EELS). A representative STEM image of a nanosheet at the edge region is shown in figure 3A. EEL spectra were recorded from the same sample region to form a map, with the intensity of each pixel in the map (ranging from black to yellow/white) corresponding to the integrated intensity of the oxygen K-edge in the pixel location. From this data, an oxygen content map could be constructed from the same sample region (Fig. 3B) as shown in STEM image (Fig.3A). Hence, the map is color-coded and shows increasing oxygen content from black/blue (no/low oxygen) to green to red to yellow to white. It is clear that the oxides reside mostly near the edges of the nanosheets and at step edges throughout the nanosheets. The EEL spectra corresponding to regions with different oxygen content are displayed in figure $3 \mathrm{C}$.

\section{Size selection}

A great advantage of liquid exfoliation is not only that nanosheets are processable from liquids, but that they can also be size-selected using well established techniques by controlled centrifugation. ${ }^{37,38,40}$ This is extremely important considering that potential applications often require control over both thickness and lateral dimensions. To demonstrate this, we have performed size-selection by controlled centrifugation with increasing centrifugation velocity in consecutive steps (see methods). This enables the production of nanosheet dispersions with varying mean sizes from the same stock dispersion in large quantities. By this procedure, five different sizes were produced and subjected to detailed characterization. For the main manuscript we focused on three different dispersions. Further data can be found in the supporting information. Displayed in figure 3A-C are TEM length histograms and representative images of three different size-selected $\mathrm{GaS}$ dispersions in IPA (data for all five sizes is given in the SI Fig. S5). The mean lateral dimensions were determined from the statistical TEM analysis yielding TEM mean lengths $<\mathrm{L}>$ ranging from $130-405 \mathrm{~nm}$. 
Since it has previously been reported that optical extinction, absorbance and scattering spectra change as a function of size, the optical response was measured for each dispersion. ${ }^{37}$, ${ }^{40}$ Any systematic spectral changes can be very valuable as - once calibrated - they can be used to establish metrics to potentially quantify both lateral dimensions and thickness of the liquid exfoliated nanosheets. ${ }^{37}$ Optical extinction (normalized to the local minimum), absorbance (normalized to the local minimum) and scattering (normalized to the local minimum in extinction) spectra of the size-selected GaS are displayed in figure 3D-F (plots showing the unnormalized coefficient spectra can be found in the SI, figure S12). All exhibit well defined and systematic changes as a function of size which will be analyzed below. Of greatest interest are the absorbance spectra (figure $3 \mathrm{E}$ ), particularly in comparison to the extinction spectra (figure 3D). As mentioned above, the extinction spectra are almost featureless. However, GaS is known to display excitonic transitions at $\sim 410 \mathrm{~nm}$ (A-exciton, direct transition at the $\Gamma$-point) and $\sim 315 \mathrm{~nm}$ (B-exciton, transition at the M-point). ${ }^{84}$ While the B-exciton is discernible in the extinction spectra as the local maximum, the A-excitonic transitions are invisible due to masking by the scattering background. However, in the absorbance spectra, both excitons can be clearly resolved. In addition, very weak features at higher wavelength are discernible in the absorbance spectra (inset figure $3 \mathrm{E}$ ) which may stem from defects. ${ }^{85}$ However, these are widely invariant with size so that we suggest they are not edge or exfoliation induced.

In addition to quantifying lateral dimensions by statistical TEM, we have determined the thickness of the exfoliated GaS nanosheets using atomic force microscopy (AFM) after deposition onto $\mathrm{Si} / \mathrm{SiO}_{2}$ wafers (see methods). We find reasonably thin nanosheets similar in appearance to the TEM images (figure $3 \mathrm{G}$ inset). Since the direct measurement of the number of layers, $\mathrm{N}$, in the case of liquid exfoliated nanomaterials is complicated by both solvent and nanosheet contributing to the apparent AFM thickness, we have used previously elaborated step height analysis ${ }^{6,37,40}$ to convert the thickness to the number of layers (SI figure S6). The resultant $\mathrm{N}$ histogram of the GaS sample with mean length of $130 \mathrm{~nm}$ is displayed in figure $3 \mathrm{G}$ showing that the nanosheets are reasonably well exfoliated with mean $\mathrm{N}$ of 10 layers. Histograms and representative images of the other sizes are shown in the SI figure S7.

However, before being certain about conversion of apparent thickness to number of layers, it needs to be ensured that only individually deposited nanosheets are taken into account. To test this, we plot the mean AFM length of the counted nanosheets as a function of the TEM length in figure S8A, finding very good agreement. Furthermore, we can use our previous 
knowledge on other liquid exfoliated 2D materials that has shown a square root dependence of $\mathrm{N}$ as function of nanosheet area. ${ }^{37,39}$ As demonstrated by figure $3 \mathrm{H}$ (and Fig. S8B), the same behavior is observed in the case of GaS strongly supporting the accuracy of the AFM number of layer determination.

We furthermore note that the structural integrity of the size-selected nanosheets was confirmed by Raman and XPS (see SI figure S9-11) which gave similar results for both nonsize-selected standard dispersions and the size selected nanosheets described in figure 3 . This shows the nanosheet spectroscopic properties other than the optical response to be generally independent of size in the regime our nanosheets are produced. For example, we do not observe layer-number induced shifts in the GaS Raman spectra as previously reported. ${ }^{75}$ However, this is not surprising, as these the Raman spectrum of $\mathrm{GaS}$ is only sensitive to layer numbers of $<5$ and the majority of our GaS nanosheets have thicknesses >5 layers.

\section{Spectroscopic metrics to determine size and concentration}

The precise quantification of both $\mathrm{N}$ and $\mathrm{L}$ as described above is important, as it provides the foundation to establish spectroscopic metrics based on extinction, absorbance and scattering spectra. In the following, we show a number of these metrics which can be used to determine information regarding $\mathrm{GaS}$ nanosheet size and thickness from optical measurements.

As mentioned above, the scattering spectra are sensitive to the nanosheet lateral size. Previous work has shown that, in the high wavelength regime, where the absorbance is negligible, the scattering exponent scales as a power law with wavelength: $\sigma \propto \lambda^{-n}$, where $n$ is the size-dependent scattering exponent which can also be used as a potential metric to quantify nanosheet length. ${ }^{4,37}$ To test whether such a metric can be established for liquidexfoliated GaS, we plot the long wavelength scattering exponent, $n$, as a function of mean nanosheet length (as measured by TEM) in figure 4A. Importantly, since absorbance of GaS is negligible at high wavelength, we obtain the same result extracting $n$ from either extinction or scattering spectra, implying that measurement in an integrating sphere is not essential to determine $\langle\mathrm{L}\rangle$. We find a roughly linear relationship (valid in this size range only) between $<\mathrm{L}>$ and $n$ which allows us to use the scattering coefficient to determine the mean length of the $\mathrm{GaS}$ nanosheets in the dispersion according to equation (2).

$$
\langle L(\mu \mathrm{m})\rangle=0.67-0.14 n
$$


Recent work has also shown that for nanosheet dispersions, the shape of the absorption spectra is sensitive to nanosheet lateral size due to edge effects. ${ }^{37}$ These spectral changes in liquid-exfoliated 2D nanomaterials can be expressed as ratios of absorbance (or extinction) at two wavelengths. These intensity ratios contain nanosheet length information due to differences in electronic properties at nanosheet edge and center. ${ }^{37}$ This effect can clearly be seen in the case of GaS when plotting the ratio of absorbance at the A-exciton / local minimum at $290 \mathrm{~nm}\left(A_{420 \mathrm{~nm}} / A_{290 \mathrm{~nm}}\right)$ as a function of $\langle\mathrm{L}\rangle$ (Fig. 4B). This plot shows a well-defined relationship allowing us to link mean nanosheet length to $A_{420 \mathrm{~nm}} / A_{290 \mathrm{~nm}}$ according to equation (3).

$$
\langle L(\mu \mathrm{m})\rangle=0.93\left(A_{420 \mathrm{~nm}} / A_{290 \mathrm{~nm}}\right)^{0.44}
$$

We note that while this shows the validity of the approach, $\langle\mathrm{L}\rangle$ determination from the scattering exponent in optical extinction spectra is more straight-forward, especially because the integrating sphere is not required.

While the determination of $\langle\mathrm{L}\rangle$ is useful, for most applications, it is critical to control concentration/mass of the nanosheets. An accurate determination of the extinction/absorbance coefficient is therefore required. Since the spectra change as function of size, extinction and absorbance coefficients are expected to changes as well (as observed for $\left.\mathrm{MoS}_{2}\right)^{37}$. We therefore also analyze extinction $\varepsilon$, absorbance $\alpha$ and scattering $\sigma$ coefficient spectra (SI Fig. S12). The GaS nanosheet concentration in each case was determined by filtration and weighing. Depending on the wavelength, the coefficients vary significantly as a function of nanosheet size (Fig S12-13). However, we observe only minor changes in the extinction coefficient at $365 \mathrm{~nm}$ over a wide range of size from 100- $300 \mathrm{~nm}$ (Fig. 4C) making this an ideal wavelength for estimation of the concentration from the extinction spectra using $\varepsilon_{365 \mathrm{~nm}}=3465 \mathrm{Lg}^{-1} \mathrm{~m}^{-1}$. Since mean nanosheet lengths from a single centrifugation step are typically below $300 \mathrm{~nm}$, we have used the approximate extinction coefficient for our process optimization (as described above). If the nanosheet size is $>300 \mathrm{~nm}$ (where $\langle\mathrm{L}>$ can be determined by equation 2 or 3 ), then the extinction coefficient can be found from an empirical fit of the data:

$$
\varepsilon_{365 n m}\left(\mathrm{Lg}^{-1} \mathrm{~m}^{-1}\right)=3465-21 \times e^{\langle L(\mu \mathrm{m})\rangle / 0.128}
$$

In addition to determination of length and concentration from the optical spectra, it would also be useful to have a metric to assess the mean number of layers. In case of $\mathrm{MoS}_{2}$, the 
energy/wavelength of the A-exciton provided such a metric due to confinement effects. ${ }^{37}$ In the case of GaS, we correlate the mean nanosheet thickness (as measured from AFM) with the peak position of the B-exciton from the absorbance spectra, $\lambda_{\mathrm{B}}$. We used the B-exciton rather than the A-exciton in this case, as the intensity of the A-exciton is comparatively weak for all GaS sizes. Shown in figure $4 \mathrm{D}$ is a plot of $\lambda_{\mathrm{B}}$ versus $\langle N\rangle$ with the correlation being clear. By applying an empirical fit, we find the mean number of layers of the liquid exfoliated $\mathrm{GaS}$ nanosheets can be determined from the peak position of the B-exciton in the absorbance spectra, $\lambda_{\mathrm{B}}$, according to equation (5)

$$
\langle N\rangle=2.24 \times 10^{-26} \times e^{\lambda_{B}(n m) / 11.5}
$$

A similar metric from the extinction spectra is presented in the SI (Fig. S14). However, we note that the position of the B-exciton does not tend to a constant value which might be associated with bulk for nanosheets thicker than $\sim 10-20$ layers as might be expected. The reasons for this are unclear and will be the subject of a future study.

The presented quantitative in situ spectroscopic metrics to determine $\langle\mathrm{L}\rangle,\langle\mathrm{N}\rangle$ and concentration of liquid exfoliated GaS underline the strength of LPE, as these will be extremely useful to prepare dispersions with known dimensions and concentrations to test in applications.

\section{Hydrogen evolution catalysis}

The size metrics and precise concentration control described above facilitates the use of liquid-exfoliated GaS nanosheets in applications where lateral nanosheet size is important. Such an application may be the hydrogen evolution reaction (HER). It is known that edge sulfur atoms in $\mathrm{MoS}_{2}$ nanosheets are involved in the electrocatalysis of hydrogen production. ${ }^{86,87} \mathrm{We}$ hypothesized that GaS may also have catalytically active edge sites. If this were to be the case, liquid exfoliated $\mathrm{GaS}$ nanosheets should catalyze $\mathrm{H}_{2}$ production with the rate of production increasing as the nanosheet size decreases. To test whether this was the case, we size-selected a $\mathrm{GaS}$ stock dispersion according to the procedure used throughout this manuscript to give three size selected dispersions. The mean nanosheet lengths were determined from the scattering exponent to be 450, 280 and $180 \mathrm{~nm}$, respectively. The dispersions were vacuumfiltered to give thin films $\left(\sim 0.65 \mathrm{mg} / \mathrm{cm}^{2}\right)$ which were transferred onto pyrolytic carbon (PyC) coated $\mathrm{Si} / \mathrm{SiO}_{2}$ substrates (Fig. S15) and characterized for hydrogen evolution catalysis in a three electrode electrochemical work station (see methods). 
The current density versus potential relative to reversible hydrogen electrode (polarization curves) for small, medium and large nanosheets are shown in figure 5A. Clear size-dependence on hydrogen production is observed with small nanosheets performing much better than larger ones. This suggests that catalytic sites active in $\mathrm{GaS}$ also reside at edges as found for other $2 \mathrm{D}$ materials. ${ }^{86,87}$ For example, as the nanosheet size is reduced from 450 to 280 to $180 \mathrm{~nm}$, the onset potential (the potential where $\mathrm{J}=1 \mathrm{~mA} / \mathrm{cm}^{2}$ ) decreases from 0.62 to 0.53 to $0.48 \mathrm{~V}$ while the current density at $0.6 \mathrm{~V}$ increases from 0.6 to 6 to $22 \mathrm{~mA} / \mathrm{cm}^{2}$ (see figure S16 and table S2). We note that, while we observe an increased content of oxides in the vicinity of the edges according to EELS (Fig. 2), this does not necessarily affect the catalytic reaction, as it was proposed that only the very outer rim of atoms are catalytically active. ${ }^{86,87}$ In addition, we do not see a decrease in catalytic performance after repeated measurement of the polarization curves (Fig. S17) and do not observe a significantly increased oxide content according to XPS after this procedure (Fig. S18).

Tafel plots of overpotential versus current density (figure 5B) show the expected behavior in the linear regime with Tafel slopes between 106 and $85 \mathrm{mV} / \mathrm{dec}$. These slopes are smaller than the values of $\sim 120 \mathrm{mV} / \mathrm{dec}$ usually found for the $2 \mathrm{H}$-polytype ${ }^{45,88}$ of $\mathrm{MoS}_{2}$ and indicate the rate limiting step may be different in $\mathrm{GaS}$ nanosheets. However, the exchange current densities were $<2.5 \times 10^{-6} \mathrm{~mA} / \mathrm{cm}^{2}$ for all three $\mathrm{GaS}$ samples, much smaller than is usually found for $2 \mathrm{H}-\mathrm{MoS}_{2}$. In addition, the onset potential was at least $480 \mathrm{mV}$ for the samples studied here, considerably larger than the values of $\sim 300 \mathrm{mV}$ usually found for $2 \mathrm{H} \mathrm{MoS} 2 .{ }^{45,88}$

Thus, at first glance GaS might appear to be a poor catalyst for hydrogen evolution in contrast to $\mathrm{MoS}_{2}$ which is commonly accepted as a promising material. This is due to its relatively high onset potential and low exchange current densities. However, the lower Tafel slope may offer some compensation for these deficiencies. To test this, we produced $2 \mathrm{H} \mathrm{MoS} 2$ nanosheets by liquid exfoliation and size selection using previously described procedures. ${ }^{37}$ We produced a film with near identical mass $\left(\sim 0.7 \mathrm{mg} / \mathrm{cm}^{2}\right)$ to the $\mathrm{GaS}$ samples and slightly smaller nanosheet size $(\mathrm{L}=120 \mathrm{~nm})$. Polarization curves for LPE GaS and $2 \mathrm{H}-\mathrm{MoS}_{2}$ are compared in Figure 5C. It is apparent that, although $\mathrm{GaS}$ requires large overpotentials to initiate the hydrogen evolution, its smaller Tafel slope causes a more rapid increase of the current with potential. In fact, both materials reach the same current density at $0.6 \mathrm{~V}$, above which $\mathrm{GaS}$ dominates. This suggests that GaS can indeed be regarded as potentially attractive hydrogen 
evolution catalyst, especially if problems associated with the low conductivity could be overcome for example by addition of nanotubes. ${ }^{89}$

\section{Conclusion}

In conclusion, we have demonstrated that layered III-VI semiconductors such as GaS can be exfoliated in appropriate solvents by sonication. Dispersibility can be well described in the framework of solution thermodynamics. While a number of solvents can be used to exfoliate GaS, we have focused on 2-propanol due to its low toxicity and boiling point. We have fully optimized sonication and centrifugation conditions to yield stable dispersions with typical concentrations of $0.2 \mathrm{~g} / \mathrm{L}$. Raman, XPS and high resolution STEM imaging show the exfoliated nanosheets to be widely structurally perfect and free of defects except for a higher oxygen content in the vicinity of edges as shown by EELS.

We utilized one advantage of liquid exfoliation techniques and performed size-selection by controlled centrifugation. This allowed us to produce liquid-exfoliated $\mathrm{GaS}$ nanosheet dispersions with mean lateral dimensions ranging from $\sim 100 \mathrm{~nm}$ to $>400 \mathrm{~nm}$ and mean number of layers from 10-40 as quantified by statistical TEM and AFM analysis. Importantly, we found optical extinction, absorbance and scattering spectra to vary strongly as a function of size and thickness. This enabled us to establish quantitative spectroscopic metrics to accurately determine mean length, thickness and concentration of the dispersion.

We subsequently used these metrics to produce nanosheet dispersions with known concentration and lateral dimensions that were used as electrodes and tested for hydrogen evolution catalysis. We find a clear size effect with smaller nanosheets performing much better than larger nanosheets. This suggests that catalytic sites are located at the edges of the nanosheets. Even though onset potentials are typically larger than for $\mathrm{MoS}_{2}$ of similar lateral dimensions, the lower Tafel slopes make GaS an attractive material for hydrogen evolution, in particular in combination with other catalysts that exhibit lower onset potential.

We believe these results are general and that it should be possible to transfer these procedures almost exactly for the exfoliation of other III-VI layered semiconductors such as InS and GaSe. This will open up this whole family of 2D materials for exploitation. In addition, we think it is important to note that this paper describes using LPE to exfoliate a member $(\mathrm{GaS})$ of yet another family of layered compounds (III-VI layered semiconductors). This underlines 
the generality and versatility of this method. We believe that many as yet untested layered materials will be exfoliated using such procedures. 


\section{Materials and Methods}

\section{Materials}

Gallium sulfide powder (99.999\% Ga-S-05-P) was purchased from American Elements. All solvents were purchased from Sigma Aldrich at the highest available purity with all being $>99 \%$.

\section{Production of GaS nanosheets}

Gallium sulfide powder was sonicated in a solvent using an ultrasonic bath (P30 H Ultrasonic from Fischer scientific). The sonication was performed with an amplitude of 100\% and a frequency of $37 \mathrm{kHz}$ in $50 \mathrm{~mL}$ plastic centrifuge tubes. The water in the sonic bath was cooled by a water cooling system to $20-30{ }^{\circ} \mathrm{C}$ (depending on length of sonication) enabled by cold water being pumped through piping which was wrapped around the interior of the bath. Once sonicated, the dispersion was centrifuged in a Hettich Mikro 220R centrifuge with a fixed-angle rotor 1060 (NB: For this centrifuge, rpm are related to $g$-force via $\mathrm{RCF}=106.4 f^{2}$ where $f$ is the rotation rate in $\mathrm{krpm}$ ). The top $60 \%$ was then taken from the centrifuged dispersion (supernatant) for analysis and the sediment was discarded. For the solvent screening, $1 \mathrm{~g} / \mathrm{L}$ of $\mathrm{GaS}$ was sonicated in $20 \mathrm{~mL}$ of each solvent for 6 hours and centrifuged for $180 \mathrm{~min}$ at $2.5 \mathrm{krpm}$. The supernatant was decanted and the absorption and extinction were measured in a $4 \mathrm{~mm}$ path length cuvette using a Perkin Elmer Lambda 650 spectrometer (details see below). The following solvents were used: Isopropanol $\left(\delta_{\mathrm{s}}=23.6 \mathrm{MPa}^{1 / 2}\right), \mathrm{N}$-methyl-2pyrrolidone $\left(\delta s=23 \mathrm{MPa}^{1 / 2}\right)$, methanol $\left(\delta \mathrm{s}=29.6 \mathrm{MPa}^{1 / 2}\right)$, chloroform $\left(\delta \mathrm{s}=19 \mathrm{MPa}^{1 / 2}\right), \mathrm{N}-$ cyclohexyl-2-pyrrolidone $\quad\left(\delta_{\mathrm{s}}=20.5 \quad \mathrm{MPa}^{1 / 2}\right)$, dimethylformamide $\quad\left(\delta_{\mathrm{s}}=24.9 \quad \mathrm{MPa}^{1 / 2}\right)$, cyclopentane $\left(\delta s=16.5 \mathrm{MPa}^{1 / 2}\right)$, heptane $\left(\delta \mathrm{s}=15.3 \mathrm{MPa}^{1 / 2}\right)$, hexane $\left(\delta \mathrm{s}=14.9 \mathrm{MPa}^{1 / 2}\right)$, pentane $\left(\delta \mathrm{s}=14.4 \mathrm{MPa}^{1 / 2}\right)$, acetone $\left(\delta \mathrm{s}=19.9 \mathrm{MPa}^{1 / 2}\right)$, acetonitrile $\left(\delta \mathrm{s}=24.3 \mathrm{MPa}^{1 / 2}\right), 1,3$-dioxolane $\left(\delta \mathrm{s}=21.4 \mathrm{MPa}^{1 / 2}\right)$, benzonitrile $\left(\delta \mathrm{s}=22.5 \mathrm{MPa}^{1 / 2}\right)$, isopropoxyethanol $\left(\delta \mathrm{s}=21.4 \mathrm{MPa}^{1 / 2}\right)$. The final, optimized exfoliation was performed as follows: $45 \mathrm{~g} / \mathrm{L}$ of $\mathrm{GaS}$ were sonicated for 6 hours in isopropanol in $20 \mathrm{~mL}$ vials and then centrifuged for $180 \mathrm{~min}$ at $2.5 \mathrm{krpm}$.

\section{Size selection}

We used controlled centrifugation with subsequently increasing rotation speeds as previously reported. ${ }^{6,40} 10 \mathrm{~g} / \mathrm{L} \mathrm{GaS}$ in $90 \mathrm{~mL}$ of isopropanol was sonicated for 6 hours in three centrifuge tubes. The $90 \mathrm{~mL}$ of sonicated dispersion was centrifuged at $0.5 \mathrm{krpm}$ for $60 \mathrm{~min}$. The sediment was discarded and the supernatant was centrifuged again at $1 \mathrm{krpm}$ for $60 \mathrm{~min}$. 
The sediment after this centrifugation step was redispersed in fresh IPA ( 5 min bath sonication) producing the largest size. The supernatant after the $1 \mathrm{krpm}$ centrifugation step was centrifuged at $1.5 \mathrm{krpm}$ for $60 \mathrm{~min}$ producing the second largest size in the redispersed sediment. These steps were repeated in further increments of $2 \mathrm{krpm}, 2.5 \mathrm{krpm}$ and $3 \mathrm{krpm}$ thus producing 5 sizes.

\section{Characterization and equipment}

Optical extinction and absorbance was measured on a Perkin Elmer 650 spectrometer in quartz cuvettes with a path length of $0.4 \mathrm{~cm}$. To distinguish between contributions from scattering and absorbance to the extinction spectra, dispersions were measured in an integrating sphere using a home-built sample holder to place the cuvette in the center of the sphere (NB cuvettes need to be transparent to all sides and correct positioning is important). The absorbance spectrum is obtained from the measurement inside the sphere. A second measurement on each dispersion was performed outside the sphere in the standard configuration to obtain the extinction spectrum. This allows calculation of the scattering spectrum (extinction minus absorbance).

Low-resolution bright field transmission electron microscopy imaging was performed using a JEOL 2100, operated at $200 \mathrm{kV}$. Holey carbon grids (400 mesh) were purchased from Agar Scientific and prepared by diluting a dispersion to a low concentration and drop casting onto a grid placed on a filter membrane to wick away excess solvent. Statistical analysis was performed of the flake dimensions by measuring the longest axis of the nanosheet and assigning it as "length", L. STEM imaging and STEM EELS was carried out on a Nion UltraSTEM100 aberration-corrected dedicated STEM, equipped with a Gatan Enfina electron energy loss (EEL) spectrometer, at the SuperSTEM Laboratory in Daresbury, UK. The microscope was operated at an acceleration voltage of $60 \mathrm{kV}$.

EELS were acquired with a convergence semi-angle of $32 \mathrm{mrad}$, a collection semiangle of approximately $37 \mathrm{mrad}$, a dispersion of $0.5 \mathrm{eV} / \mathrm{channel}$ and an energy resolution over vacuum of $0.3 \mathrm{eV}$. STEM EELS maps were acquired with $0.1 \mathrm{~s}$ acquisition time per spectra per pixel. Subsequently, the spectra were aligned to the Oxygen K-edge. The spectra shown here are representative of a set of 10 STEM EELS maps that were acquired over similar regions. 
Atomic force microscopy (AFM) was carried out on a Veeco Nanoscope-IIIa (Digital Instruments) system equipped with a E-head (13 $\mu \mathrm{m}$ scanner) in tapping mode after depositing a drop of the dispersion $(10 \mu \mathrm{L})$ on a pre-heated $\left(120^{\circ} \mathrm{C}\right) \mathrm{Si} / \mathrm{SiO}_{2}$ wafer with an oxide layer of $300 \mathrm{~nm}$. Typical image sizes were $3-10 \mu \mathrm{m}$ at scan rates of $0.4-0.6 \mathrm{~Hz}$.

Raman spectroscopy was performed using a WITec alpha 300 with $532 \mathrm{~nm}$ excitation laser in air under ambient conditions. The Raman emission was collected by an Olympus 100x objective (N.A. $=0.8$ ) and dispersed by 600 lines $\mathrm{mm}^{-1}$ gratings. The laser energy was kept below $0.2 \mathrm{~mW}$. The mean of 20 spectra is displayed.

$\mathrm{X}$-ray Photoelectron Spectroscopy was performed under ultra-high vacuum conditions $\left(<5 \times 10^{-10}\right.$ mbar) using monochromated Al Ka X-rays from an Omicron XM1000 MkII X-ray source and an Omicron EA125 energy analyzer. The analyzer pass energy was set to $100 \mathrm{eV}$ for survey and $20 \mathrm{eV}$ for core-level spectra, yielding a maximum energy resolution of $\sim 0.65$ $\mathrm{eV}$. An electron flood gun was used for charge compensation and the binding energy scale was referenced to the adventitious carbon 1s core-level at $284.8 \mathrm{eV}$. After subtraction of a Shirley background, the core-level spectra were fitted with Gaussian-Lorentzian line shapes. Samples were prepared by vacuum-filtering the dispersions using porous cellulose filter membranes (MF-Millipore membrane, mixed cellulose esters, hydrophilic, $0.025 \mu \mathrm{m}, 47 \mathrm{~mm}$ ) to give thin films.

Scanning Electron Microscopy was performed with a Carl Zeiss Ultra SEM operating at $2 \mathrm{kV}$. Images were acquired using the secondary electron detector. Powder XRD was performed using a Siemens D500 X-ray Diffractometer equipped with a $\mathrm{Cu} \mathrm{K} \alpha$ emission source $(\lambda=1.54056 \AA)$ filtered through a graphite monochromator, at ambient temperature.

\section{Hydrogen evolution catalysis}

Dispersions of GaS in IPA were vacuum-filtered using porous cellulose filter membranes (MF-Millipore membrane, mixed cellulose esters, hydrophilic, $0.025 \mu \mathrm{m}$ ) to give uniform thin films. The deposited films were then cut into pieces $\left(0.65 \mathrm{~cm}^{2}\right)$ and transferred on

PyC electrodes using heat and pressure as described previously. ${ }^{24,} 90$ The cellulose filter membrane was then removed by treatment with acetone vapor and subsequent acetone liquid baths followed by an isopropanol rinse to remove the acetone residue. The mass per area for all samples was approximately $0.65 \mathrm{mg} / \mathrm{cm}^{2}$. 
Pyrolytic carbon was grown by chemical vapor deposition (CVD) from an acetylene feedstock at $950{ }^{\circ} \mathrm{C}$ for 30 minutes to a thickness of $300-400 \mathrm{~nm}$ on $300 \mathrm{~nm}$ thermal $\mathrm{SiO}_{2}$ on Si substrates in a hot wall quartz tube furnace as previously reported. ${ }^{91}$

Electrochemical measurements were carried out to evaluate the performance of $\mathrm{GaS}$ on PyC films as electrodes for HER. The measurements were performed in $0.5 \mathrm{M} \mathrm{H}_{2} \mathrm{SO}_{4}$ solution using a three-electrode electrochemical cell, with a reversible hydrogen electrode (RHE) reference electrode and a graphite rod counter electrode. Electrochemical tests consisted of linear sweep voltammetry and electrochemical impedance spectroscopy using a Gamry Reference 3000 potentiostat. Before each test, samples were conditioned at a given voltage for $3 \mathrm{~min}$. The electrocatalysis was measured using linear sweeping from $0 \mathrm{~V}$ to $-0.8 \mathrm{~V}$ (vs. RHE) with a scan rate of $5 \mathrm{mV} / \mathrm{S}$. The $\mathrm{AC}$ impedance was measured within the frequency range of 0.1 to $10 \mathrm{mHz}$ with perturbation voltage amplitude of $10 \mathrm{mV}$. The equivalent series resistance of the system was measured by impedance spectroscopy from the high frequency intercept with the real impedance axis and all the data were corrected by $\mathrm{iR}$ compensation.

Supporting Information Available: This includes more detailed descriptions of the basic characterization, size selection, optical characterization and hydrogen evolution. This information is available free of charge via the Internet at http://pubs.acs.org/.

\section{Acknowledgement}

The research leading to these results has received funding from the European Union Seventh Framework Programme under grant agreement $n^{\circ} 604391$ Graphene Flagship. We have also received support from the Science Foundation Ireland (SFI) funded centre AMBER (SFI/12/RC/2278). In addition, JNC acknowledges the European Research Council (SEMANTICS) and SFI (11/PI/1087) for financial support. CB acknowledges the German research foundation DFG (BA 4856/1-1). HCN, EMcG, AS-A and VN acknowledge ERC 2DNanoCaps, SFI PIYRA and FP7 MoWSeS. GSD, NCB and SW acknowledge SFI for PI_10/IN.1/I3030. NMcE acknowledges SFI (14/TIDA/2329). STEM experiments were performed at SuperSTEM, the EPSRC UK national facility for aberration-corrected STEM. 

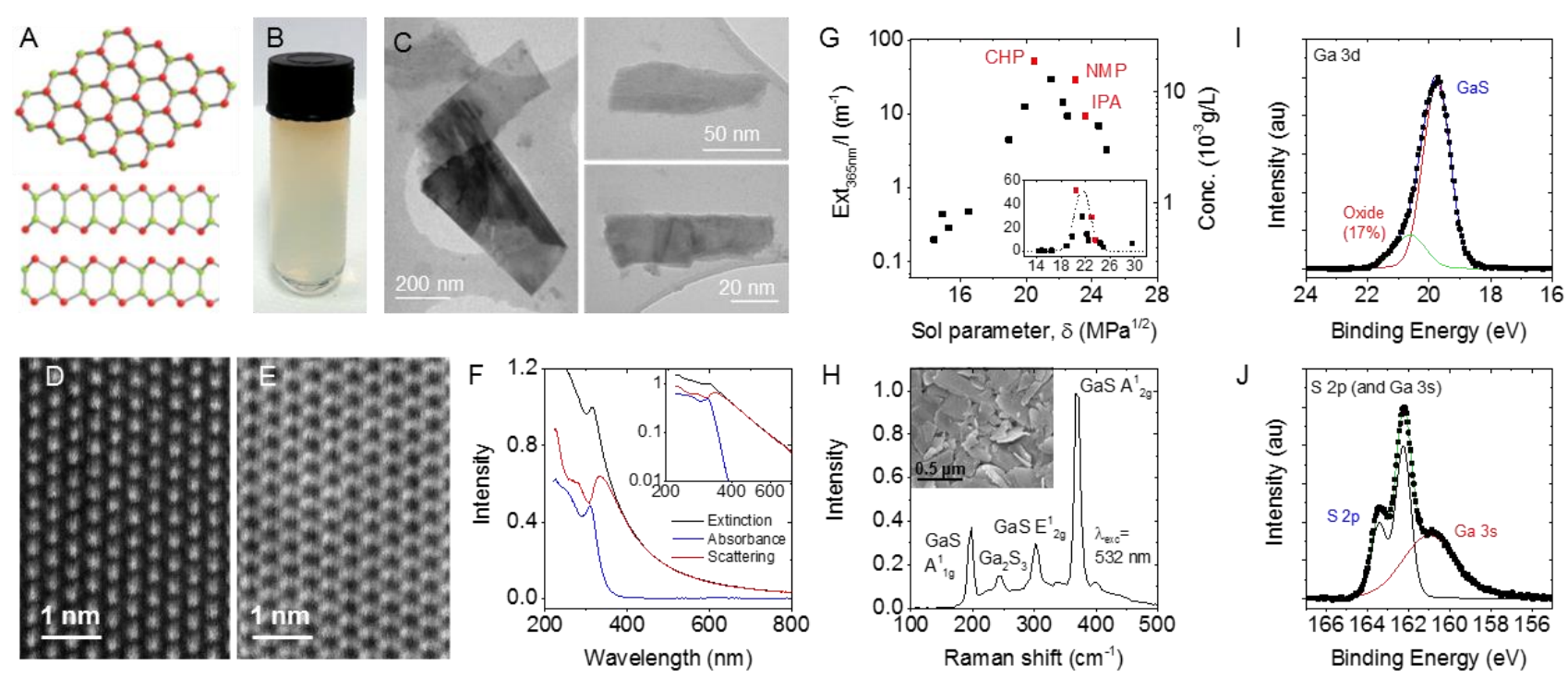

Figure 1: A) Structure of gallium sulfide, GaS, in top and side view. Red: S, Green: Ga. B) Photograph of a typical dispersion (in 2-propanol). C) Representative low-resolution bright field TEM images of GaS nanosheets exfoliated in IPA. D) Bright-field scanning transmission TEM image and E) high angle annular dark field (HAADF) STEM image of GaS showing the intact lattice. F) Optical extinction, absorbance and scattering spectra of a typical GaS nanosheet dispersion in IPA. G) Dispersed concentration of GaS expressed as optical extinction at $365 \mathrm{~nm}$ divided by cell length plotted as a function of the Hildebrand solubility parameter $\delta$ s of the solvent. The concentration (right axis) is estimated by the extinction coefficient at 365 $\mathrm{nm}$. The dashed line in the inset is a fit to eq 1 . The dispersions in panel $\mathrm{G}$ were prepared with initial $\mathrm{GaS}$ concentration $\mathrm{C}_{\mathrm{i}}=1 \mathrm{~g} / \mathrm{L}$ and each was sonicated for $\mathrm{t}_{\mathrm{s}}=6 \mathrm{~h}$ and centrifuged at $f=2$ $\mathrm{krpm}$ for $\mathrm{t}_{\mathrm{CF}}=180 \mathrm{~min} . \mathrm{H}$ ) Raman spectrum (excitation wavelength $532 \mathrm{~nm}$, mean of 20 spectra) of the filtered standard dispersion (IPA, $\mathrm{C}_{\mathrm{i}}=45 \mathrm{~g} / \mathrm{L}, \mathrm{t}_{\mathrm{s}}=6 \mathrm{~h}, f=2.5 \mathrm{krpm}, \mathrm{t}_{\mathrm{CF}}=180$ min) showing the characteristic phonons of $\mathrm{GaS}$ and a small impurity contribution which we associate with $\mathrm{Ga}_{2} \mathrm{~S}_{3}$. Inset: SEM image of the film. I, J) Fitted XPS core level spectra of a filtered dispersion confirming the chemical nature of the nanosheets. I) Ga $3 \mathrm{~d}$ core level spectrum, J) S 2p core level spectrum. 

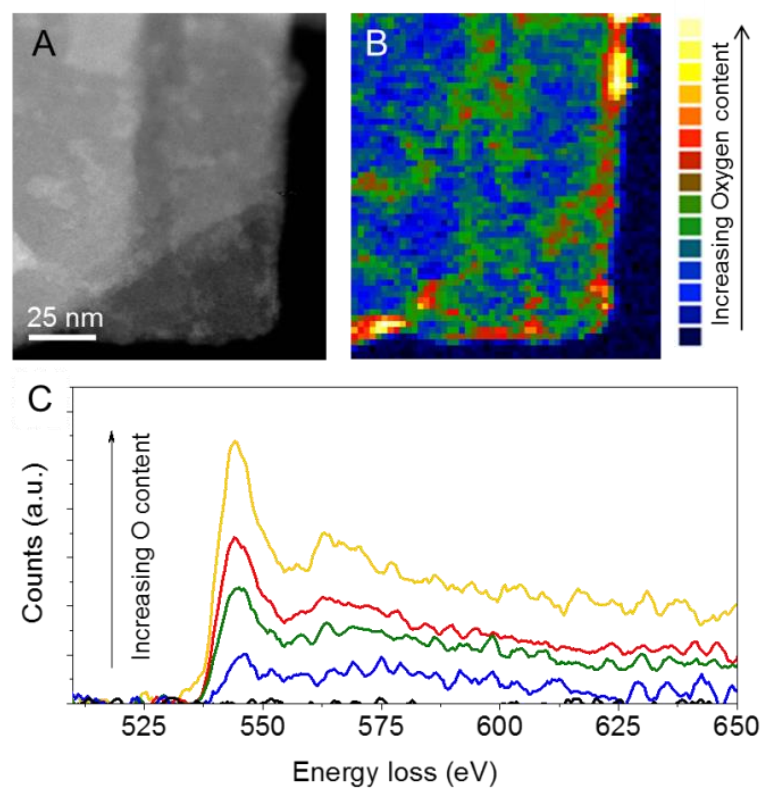

Figure 2: A) Scanning transmission electron microscopic image of a GaS nanosheet. B) Colorcoded electron energy loss (EEL) map of the same region. Each pixel in the map corresponds to the integrated intensity of the energy loss signal of the oxygen K-edge after background removal to the same edge. Hence, the map shows the variation in the oxygen content across the region where increasing oxygen content is shown from black to green to red to yellow to white. The oxygen signal is most prominent on the edges of the nanosheet and at step edges in the center of the nanosheet as indicated in the figure (yellow/white regions). C) Representative EEL spectra extracted at different positions of the nanosheet, with the colors of the spectra corresponding to the colors in the map in panel B. The spectra shown in yellow represent the highest $\mathrm{O}$ content, as found near the edges of the nanosheets, and the spectra shown in black and blue representing the lowest $\mathrm{O}$ content, as found on the surfaces inside the flake and over vacuum. 

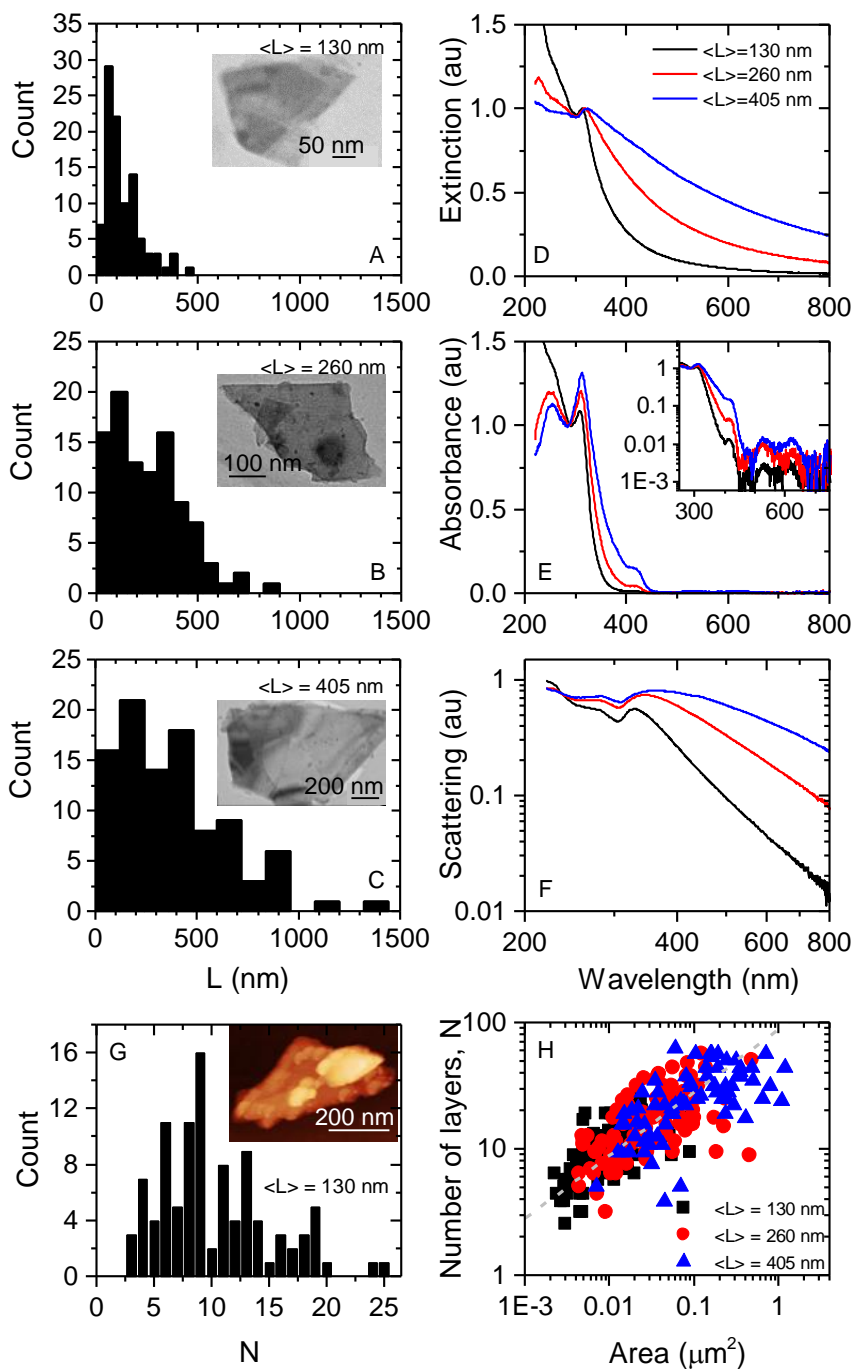

Figure 3: A-C) Length histograms and representative images of size-selected GaS in IPA as insets. A) smallest-size GaS, B) medium-sized GaS, C) large GaS. The mean length $\langle\mathrm{L}\rangle$ is shown as figure legend. Data for additional sizes are given in the SI. D-F) Optical extinction, absorbance and scattering spectra of dispersions of different sized nanosheets showing systematic changes in spectral shape. D) Extinction, E) absorbance and F) scattering. Extinction and absorbance spectra are normalized to their local minimum while scattering spectra are normalized to the minimum in the extinction spectra. The inset in E shows the absorbance on a log scale to highlight the A-exciton $(\sim 420 \mathrm{~nm})$ and the gap features. G) Typical AFM number of layers, N, histogram of smallest-size GaS in IPA drop cast onto

$\mathrm{Si} / \mathrm{SiO}_{2}$ wafers. The mean number of layers was determined from step height analysis (see SI). Inset: representative image of a nanosheet. H) Plot of number of layers per nanosheet as a function of flake area determined from AFM. The dashed line indicates $N \propto \sqrt{A}$ behavior. 

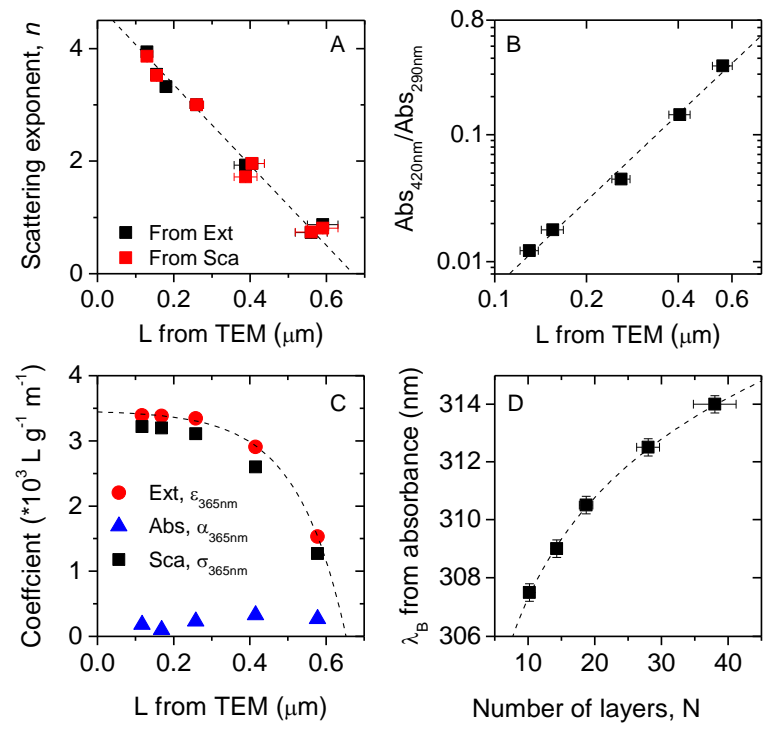

Figure 4: A) Long wavelength scattering exponent, $n$, measured from both extinction and scattering spectra, plotted versus TEM mean length, L, for size-selected GaS in IPA. B) Ratio of absorbance at $420 \mathrm{~nm}$ to that at $290 \mathrm{~nm}$, Abs420nm/Abs290nm plotted versus TEM length, L, for size selected GaS in IPA. The fit lines plotted in A and B can be used as metrics to determine $\mathrm{L}$ according to eq 2 and 3, respectively. C) Extinction, absorbance and scattering coefficients as a function of $\mathrm{L}$. The fit line can be used to find the extinction coefficient for a mean nanosheet length which in turn can be used to determine the dispersion concentration from the optical extinction spectra according to eq 4. D) Position of the B-exciton, $\lambda_{\mathrm{B}}$ (measured from the absorbance spectra), plotted versus number of monolayers per nanosheet, $\mathrm{N}$ (determined from AFM). The relation can be used as metric to determine $\mathrm{N}$ according to eq 5 . 

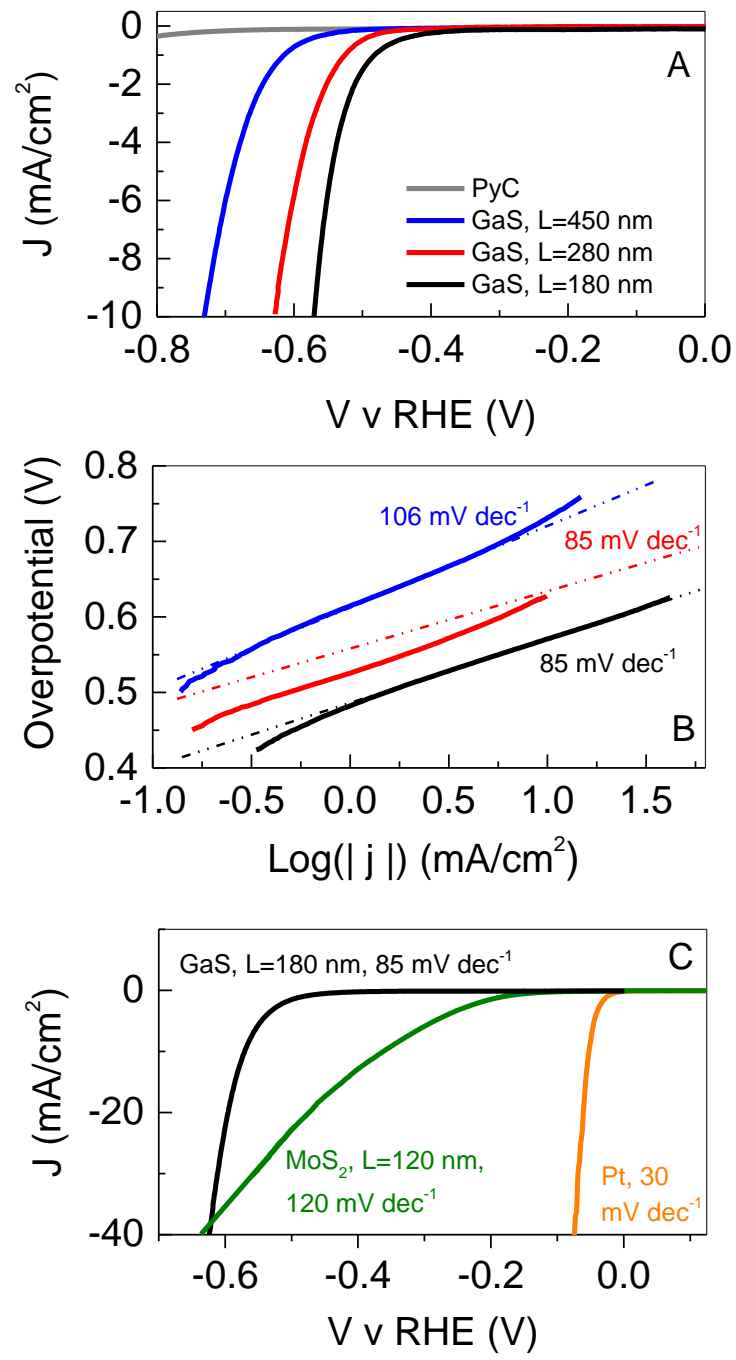

Figure 5: A) Linear sweep voltammograms $\left(5 \mathrm{mV} \mathrm{s}^{-1}\right)$ of vacuum-filtered $\mathrm{GaS}$ nanosheets transferred onto pyrolytic carbon with a reversible hydrogen (RHE) reference electrode. The electrocatalytic response towards the hydrogen-evolution reaction is compared for sizeselected GaS with three different mean sizes. A clear size-dependence is evident. Measured potentials were subjected to iR correction. Supporting electrolyte was $0.5 \mathrm{M} \mathrm{H}_{2} \mathrm{SO}_{4}$. B) Overpotential versus current density plots showing Tafel slopes expressed as $\mathrm{mV}$ per decade $\left(\mathrm{mV} \mathrm{dec}^{-1}\right)$. C) Linear sweep voltammograms of s-GaS compared to liquid-exfoliated $2 \mathrm{H}-\mathrm{MoS}_{2}$ of similar lateral dimensions and mass per area and Pt as benchmark. Tafel slopes are indicated in the figure. Despite of the high onset potential, s-GaS reaches the same current density as $\mathrm{MoS}_{2}$ at $\sim 0.6 \mathrm{eV}$ due to its smaller Tafel slope.

\section{References}


1. Du, W.; Jiang, X.; Zhu, L., J. Mater. Chem. C. 2013, 1, 10592-10606.

2. Nicolosi, V.; Chhowalla, M.; Kanatzidis, M. G.; Strano, M. S.; Coleman, J. N., Science 2013, $340,1420-+$.

3. $\quad$ Ciesielski, A.; Samori, P., Chem. Soc. Rev. 2014, 43, 381-398.

4. $\quad$ Smith, R. J.; King, P. J.; Lotya, M.; Wirtz, C.; Khan, U.; De, S.; O'Neill, A.; Duesberg, G. S.; Grunlan, J. C.; Moriarty, G.; Chen, J.; Wang, J.; Minett, A. I.; Nicolosi, V.; Coleman, J. N., Adv. Mater. 2011, 23, 3944-3948.

5. Hernandez, Y.; Nicolosi, V.; Lotya, M.; Blighe, F. M.; Sun, Z.; De, S.; McGovern, I. T.; Holland, B.; Byrne, M.; Gun'Ko, Y. K.; Boland, J. J.; Niraj, P.; Duesberg, G.; Krishnamurthy, S.; Goodhue, R.; Hutchison, J.; Scardaci, V.; Ferrari, A. C.; Coleman, J. N., Nat. Nanotechnol. 2008, 3, 563-568.

6. $\quad$ Paton, K. R.; Varrla, E.; Backes, C.; Smith, R. J.; Khan, U.; O’Neill, A.; Boland, C.; Lotya, M.; Istrate, O. M.; King, P.; Higgins, T.; Barwich, S.; May, P.; Puczkarski, P.; Ahmed, I.; Moebius, M.; Pettersson, H.; Long, E.; Coelho, J.; O’Brien, S. E.; McGuire, E. K.; Sanchez, B. M.; Duesberg, G. S.; McEvoy, N.; Pennycook, T. J.; Downing, C.; Crossley, A.; Nicolosi, V.; Coleman, J. N., Nat. Mater. 2014, 13, 624-630.

7. Varrla, E.; Paton, K. R.; Backes, C.; Harvey, A.; Smith, R. J.; McCauley, J.; Coleman, J. N., Nanoscale 2014.

8. $\quad$ Liu, L.; Shen, Z. G.; Yi, M.; Zhang, X. J.; Ma, S. L., RSC Adv. 2014, 4, 36464-36470.

9. $\quad$ Yi, M.; Shen, Z. G., Carbon 2014, 78, 622-626.

10. Hernandez, Y.; Lotya, M.; Rickard, D.; Bergin, S. D.; Coleman, J. N., Langmuir 2010, 26, 3208-3213.

11. Bourlinos, A. B.; Georgakilas, V.; Zboril, R.; Steriotis, T. A.; Stubos, A. K., Small 2009, 5, 1841-1845.

12. $\quad$ Du, W.; Lu, J.; Sun, P.; Zhu, Y.; Jiang, X., Chem. Phys. Lett. 2013, 568, 198-201.

13. Liu, W. W.; Wang, J. N., Chem. Commun. 2011, 47, 6888-6890.

14. Oyer, A. J.; Carrillo, J.-M. Y.; Hire, C. C.; Schniepp, H. C.; Asandei, A. D.; Dobrynin, A. V.; Adamson, D. H., J. Am. Chem. Soc. 2012, 134, 5018-5021.

15. Yi, M.; Shen, Z.; Ma, S.; Zhang, X., J. Nanopart. Res. 2012, 14.

16. Yi, M.; Shen, Z.; Zhang, X.; Ma, S., J. Phys. D: Appl. Phys. 2013, 46.

17. Zhou, K.-G.; Mao, N.-N.; Wang, H.-X.; Peng, Y.; Zhang, H.-L., Angew. Chem.,Int. Ed. 2011, $50,10839-10842$.

18. Bang, G. S.; Nam, K. W.; Kim, J. Y.; Shin, J.; Choi, J. W.; Choi, S.-Y., ACS Appl. Mater. Interfaces 2014, 6, 7084-7089.

19. Shmeliov, A.; Shannon, M.; Wang, P.; Kim, J. S.; Okunishi, E.; Nellist, P. D.; Dolui, K.; Sanvito, S.; Nicolosi, V., ACS Nano 2014, 8, 3690-3699.

20. $\quad$ Sun, L.; Lin, Z.; Peng, J.; Weng, J.; Huang, Y.; Luo, Z., Sci. Rep. 2014, 4.

21. Nuvoli, D.; Valentini, L.; Alzari, V.; Scognamillo, S.; Bon, S. B.; Piccinini, M.; Illescas, J.; Mariani, A., J. Mater. Chem. 2011, 21, 3428-3431.

22. Lotya, M.; Hernandez, Y.; King, P. J.; Smith, R. J.; Nicolosi, V.; Karlsson, L. S.; Blighe, F. M.; De, S.; Wang, Z.; McGovern, I. T.; Duesberg, G. S.; Coleman, J. N., J. Am. Chem. Soc. 2009, 131, 3611-3620.

23. Green, A. A.; Hersam, M. C., Nano Lett. 2009, 9, 4031-4036.

24. De, S.; King, P. J.; Lotya, M.; O'Neill, A.; Doherty, E. M.; Hernandez, Y.; Duesberg, G. S.; Coleman, J. N., Small 2010, 6, 458-464.

25. Guardia, L.; Fernandez-Merino, M. J.; Paredes, J. I.; Solis-Fernandez, P.; Villar-Rodil, S.; Martinez-Alonso, A.; Tascon, J. M. D., Carbon 2011, 49, 1653-1662.

26. Lin, S.; Shih, C.-J.; Strano, M. S.; Blankschtein, D., J. Am. Chem. Soc. 2011, 133, 12810-12823.

27. Shih, C.-J.; Paulus, G. L. C.; Wang, Q. H.; Jin, Z.; Blankschtein, D.; Strano, M. S., Langmuir 2012, 28, 8579-8586.

28. Gao, H.; Shori, S.; Chen, X.; zur Loye, H.-C.; Ploehn, H. J., J. Colloid Interface Sci. 2013, 392, 226-236. 
29. Guardia, L.; Paredes, J. I.; Rozada, R.; Villar-Rodil, S.; Martinez-Alonso, A.; Tascon, J. M. D., RSC Adv. 2014, 4, 14115-14127.

30. Samoilov, V. M.; Danilov, E. A.; Nikolaeva, A. V.; Yerpuleva, G. A.; Trofimova, N. N.; Abramchuk, S. S.; Ponkratov, K. V., Carbon 2015, 84, 38-46.

31. $\quad$ Wang, S.; Yi, M.; Shen, Z.; Zhang, X.; Ma, S., RSC Adv. 2014, 4, 25374-25378.

32. Zhang, L.; Zhang, Z.; He, C.; Dai, L.; Liu, J.; Wang, L., ACS Nano 2014, 8, 6663-6670.

33. May, P.; Khan, U.; Hughes, J. M.; Coleman, J. N., J. Phys. Chem. C 2012, 116, 11393-11400.

34. Bourlinos, A. B.; Georgakilas, V.; Zboril, R.; Steriotis, T. A.; Stubos, A. K.; Trapalis, C., Solid State Commun. 2009, 149, 2172-2176.

35. Chabot, V.; Kim, B.; Sloper, B.; Tzoganakis, C.; Yu, A., Sci. Rep. 2013, 3.

36. Quinn, M. D. J.; Ngoc Han, H.; Notley, S. M., ACS Appl. Mater. Interfaces 2013, 5, 1275112756.

37. Backes, C.; Smith, R. J.; McEvoy, N.; Berner, N. C.; McCloskey, D.; Nerl, H. C.; O’Neill, A.; King, P. J.; Higgins, T.; Hanlon, D.; Scheuschner, N.; Maultzsch, J.; Houben, L.; Duesberg, G. S.; Donegan, J. F.; Nicolosi, V.; Coleman, J. N., Nature Commun. 2014, 5, 4576.

38. Khan, U.; O'Neill, A.; Porwal, H.; May, P.; Nawaz, K.; Coleman, J. N., Carbon 2012, 50, 470475.

39. Hanlon, D.; Backes, C.; Higgins, T. M.; Hughes, M.; O’Neill, A.; King, P.; McEvoy, N.; Duesberg, G. S.; Mendoza Sanchez, B.; Pettersson, H.; Nicolosi, V.; Coleman, J. N., Chem. Mater. 2014, 26, 1751-1763.

40. Hanlon, D.; Backes, B.; Doherty, E.; Cucinotta, C. S.; Berner, N. C.; Boland, C.; Lee, K.; Lynch, L.; Gholamvand, Z.; Harvey, A.; Zhang, S.; Wang, K.; Moynihan, G.; Pokle, A.; Ramasse, Q. M.; McEvoy, N.; Blau, W. J.; Wang, J.; Sanvito, S.; O’Regan, D. D.; Duesberg, G. S.; Nicolosi, V.; Coleman, J. N., arXiv:1501.01881 2015.

41. Kang, J.; Seo, J.-W. T.; Alducin, D.; Ponce, A.; Yacaman, M. J.; Hersam, M. C., Nat Commun 2014, 5 .

42. $\quad$ Backes, C.; Berner, N. C.; Chen, X.; Lafargue, P.; LaPlace, P.; Freeley, M.; Duesberg, G. S.; Coleman, J. N.; McDonald, A. R., Angew. Chem., Int. Ed. 2015, accepted, 10.1002/anie.201409412R2.

43. Eigler, S.; Hirsch, A., Angew. Chem.,Int. Ed. 2014, 53, 7720-7738.

44. $\quad$ Backes, C.; Berner, N. C.; Chen, X.; Lafargue, P.; LaPlace, P.; Freeley, M.; Duesberg, G. S.; Coleman, J. N.; McDonald, A. R., Angew. Chem.,Int. Ed. 2015, 54, 2638-2642.

45. Voiry, D.; Salehi, M.; Silva, R.; Fujita, T.; Chen, M.; Asefa, T.; Shenoy, V. B.; Eda, G.; Chhowalla, M., Nano Lett. 2013, 13, 6222-6227.

46. Voiry, D.; Yamaguchi, H.; Li, J.; Silva, R.; Alves, D. C. B.; Fujita, T.; Chen, M.; Asefa, T.; Shenoy, V. B.; Eda, G.; Chhowalla, M., Nat. Mater. 2013, 12, 850-855.

47. Jan, R.; May, P.; Bell, A. P.; Habib, A.; Khan, U.; Coleman, J. N., Nanoscale 2014, 6, 48894895.

48. Khan, U.; May, P.; O'Neill, A.; Bell, A. P.; Boussac, E.; Martin, A.; Semple, J.; Coleman, J. N., Nanoscale 2013, 5, 581-587.

49. May, P.; Khan, U.; O'Neill, A.; Coleman, J. N., J. Mater. Chem. 2012, 22, 1278-1282.

50. Zhi, C. Y.; Bando, Y.; Tang, C. C.; Kuwahara, H.; Golberg, D., Adv. Mater. 2009, 21, 2889-+.

51. Young, R. J.; Kinloch, I. A.; Gong, L.; Novoselov, K. S., Compos. Sci. Technol. 2012, 72, 14591476.

52. Torrisi, F.; Hasan, T.; Wu, W.; Sun, Z.; Lombardo, A.; Kulmala, T. S.; Hsieh, G.-W.; Jung, S.; Bonaccorso, F.; Paul, P. J.; Chu, D.; Ferrari, A. C., ACS Nano 2012, 6, 2992-3006.

53. Finn, D. J.; Lotya, M.; Cunningham, G.; Smith, R. J.; McCloskey, D.; Donegan, J. F.; Coleman, J. N., J. Mater. Chem. C 2014, 2, 925-932.

54. Blake, P.; Brimicombe, P. D.; Nair, R. R.; Booth, T. J.; Jiang, D.; Schedin, F.; Ponomarenko, L. A.; Morozov, S. V.; Gleeson, H. F.; Hill, E. W.; Geim, A. K.; Novoselov, K. S., Nano Lett. 2008, 8, 1704-1708.

55. Cunningham, G.; Lotya, M.; Cucinotta, C. S.; Sanvito, S.; Bergin, S. D.; Menzel, R.; Shaffer, M. S. P.; Coleman, J. N., ACS Nano 2012, 6, 3468-3480.

56. Du, W. C.; Lu, J.; Sun, P. P.; Zhu, Y. Y.; Jiang, X. Q., Chem. Phys. Lett. 2013, 568, 198-201. 
57. Yasaei, P.; Kumar, B.; Foroozan, T.; Wang, C.; Asadi, M.; Tuschel, D.; Indacochea, J. E.; Klie, R. F.; Salehi-Khojin, A., Adv. Mater. 2015, DOI: 10.1002/adma.201405150.

58. Brent, J. R.; Savjani, N.; Lewis, E. A.; Haigh, S. J.; Lewis, D. J.; O'Brien, P., Chem. Commun. 2014, 50, 13338-13341.

59. Jacobs-Gedrim, R. B.; Shanmugam, M.; Jain, N.; Durcan, C. A.; Murphy, M. T.; Murray, T. M.; Matyi, R. J.; Moore, R. L.; Yu, B., ACS Nano 2013, 8, 514-521.

60. Meng, X.; He, K.; Su, D.; Zhang, X.; Sun, C.; Ren, Y.; Wang, H.-H.; Weng, W.; Trahey, L.; Canlas, C. P.; Elam, J. W., Adv. Funct. Mater. 2014, 24, 5435-5442.

61. Hu, P.; Wang, L.; Yoon, M.; Zhang, J.; Feng, W.; Wang, X.; Wen, Z.; Idrobo, J. C.; Miyamoto, Y.; Geohegan, D. B.; Xiao, K., Nano Lett. 2013, 13, 1649-1654.

62. Catalano, I. M.; Ferrara, M.; Tantalo, P., physica status solidi (a) 1973, 20, K135-K138.

63. Kipperman, A. H. M.; van der Leeden, G. A., Solid State Commun. 1968, 6, 657-662.

64. Yang, S.; Li, Y.; Wang, X.; Huo, N.; Xia, J.-B.; Li, S.-S.; Li, J., Nanoscale 2014, 6, 2582-2587.

65. Bourdon, A.; Bringuier, E.; Portella, M. T.; Vivières, M.; Piccioli, N., Phys. Rev. Lett. 1990, 65, 1925-1928.

66. De Blasi, C.; Drigo, A. V.; Micocci, G.; Tepore, A.; Mancini, A. M., J. Cryst. Growth 1989, 94, 455-458.

67. Parlak, M.; Erçelebi, Ç.; Günal, I.; Özkan, H.; Gasanly, N. M., Cryst. Res. Technol. 1996, 31, 673-678.

68. Hu, P.; Wen, Z.; Wang, L.; Tan, P.; Xiao, K., ACS Nano 2012, 6, 5988-5994.

69. Aksimentyeva, O. I.; Demchenko, P.; Savchyn, V. P.; Balitskii, O. A., Nanoscale Res. Lett. 2013, 8, 29.

70. Li, X.; Lin, M.-W.; Puretzky, A. A.; Idrobo, J. C.; Ma, C.; Chi, M.; Yoon, M.; Rouleau, C. M.; Kravchenko, I. I.; Geohegan, D. B.; Xiao, K., Sci. Rep. 2014, 4, 5497.

71. Tamalampudi, S. R.; Lu, Y.-Y.; Kumar U, R.; Sankar, R.; Liao, C.-D.; Moorthy B, K.; Cheng, C.-H.; Chou, F. C.; Chen, Y.-T., Nano Lett. 2014, 14, 2800-2806.

72. $\quad$ Eckhoff, W.; Putnam, R.; Wang, S.; Curl, R.; Tittel, F., Applied Physics B 1996, 63, 437-441.

73. Shen, G.; Chen, D.; Chen, P.-C.; Zhou, C., ACS Nano 2009, 3, 1115-1120.

74. Taylor, M. J., J. Raman Spectrosc. 1973, 1, 355-358.

75. Late, D. J.; Liu, B.; Matte, H. S. S. R.; Rao, C. N. R.; Dravid, V. P., Adv. Funct. Mater. 2012, 22, 1894-1905.

76. Yadgarov, L.; Choi, C. L.; Sedova, A.; Cohen, A.; Rosentsveig, R.; Bar-Elli, O.; Oron, D.; Dai, H.; Tenne, R., ACS Nano 2014, 8, 3575-3583.

77. O'Neill, A.; Khan, U.; Coleman, J. N., Chem. Mater. 2012, 24, 2414-2421.

78. Hughes, J. M.; Aherne, D.; Coleman, J. N., J. Appl. Polym. Sci. 2013, 127, 4483-4491.

79. Lucazeau, G.; Leroy, J., Spectrochim. Acta, Part A 1978, 34, 29-32.

80. Ho, C.-H.; Chen, H.-H., Sci. Rep. 2014, 4.

81. Castellanos-Gomez, A.; Vicarelli, L.; Prada, E.; Island, J. O.; Narasimha-Acharya, K. L.; Blanter, S. I.; Groenendijk, D. J.; Buscema, M.; Steele, G. A.; Alvarez, J. V.; Zandbergen, H. W.; Palacios, J. J.; van der Zant, H. S. J., 2D Materials 2014, 1, 025001.

82. Wood, J. D.; Wells, S. A.; Jariwala, D.; Chen, K.-S.; Cho, E.; Sangwan, V. K.; Liu, X.; Lauhon, L. J.; Marks, T. J.; Hersam, M. C., Nano Lett. 2014.

83. Li, L.; Yu, Y.; Ye, G. J.; Ge, Q.; Ou, X.; Wu, H.; Feng, D.; Chen, X. H.; Zhang, Y., Nat. Nanotechnol. 2014, 9, 372-377.

84. Ho, C. H.; Lin, S. L., J. Appl. Phys. 2006, 100, 083508.

85. Aydinli, A.; Gasanly, N. M.; Goksen, K., J. Appl. Phys. 2000, 88, 7144-7149.

86. Lassalle-Kaiser, B.; Merki, D.; Vrubel, H.; Gul, S.; Yachandra, V. K.; Hu, X.; Yano, J., J. Am. Chem. Soc. 2015, 137, 314-21.

87. Jaramillo, T. F.; Jorgensen, K. P.; Bonde, J.; Nielsen, J. H.; Horch, S.; Chorkendorff, I., Science 2007, 317, 100-102.

88. Wang, H. T.; Lu, Z. Y.; Xu, S. C.; Kong, D. S.; Cha, J. J.; Zheng, G. Y.; Hsu, P. C.; Yan, K.; Bradshaw, D.; Prinz, F. B.; Cui, Y., Proc. Natl. Acad. Sci. U. S. A. 2013, 110, 19701-19706. 
89. Higgins, T. M.; McAteer, D.; Coelho, J. C. M.; Sanchez, B. M.; Gholamvand, Z.; Moriarty, G.; McEvoy, N.; Berner, N. C.; Duesberg, G. S.; Nicolosi, V.; Coleman, J. N., ACS Nano 2014, 8, 95679579.

90. $\quad$ Wu, Z.; Chen, Z.; Du, X.; Logan, J. M.; Sippel, J.; Nikolou, M.; Kamaras, K.; Reynolds, J. R.; Tanner, D. B.; Hebard, A. F.; Rinzler, A. G., Science 2004, 305, 1273.

91. McEvoy, N.; Peltekis, N.; Kumar, S.; Rezvani, E.; Nolan, H.; Keeley, G. P.; Blau, W. J.; Duesberg, G. S., Carbon 2012, 50, 1216-1226. 
ToC fig

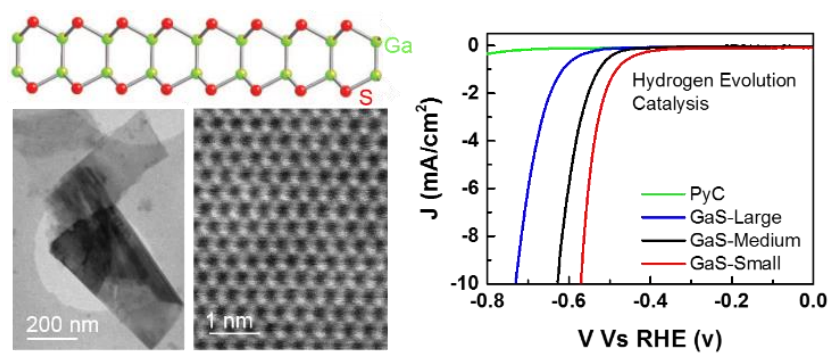

